\title{
Doppler-aided Localization of Mobile Nodes in an Underwater Distributed Antenna System
}

\author{
Patrick Carroll ${ }^{\mathrm{a}}$, Katherine Domrese ${ }^{\mathrm{a}}$, Hao Zhou ${ }^{\mathrm{b}}$, Shengli Zhou ${ }^{\mathrm{a}, *}$, Peter Willett ${ }^{\mathrm{a}}$ \\ ${ }^{a}$ Dept. of Electrical and Computer Engineering, University of Connecticut, Storrs, CT 06269, USA \\ ${ }^{b}$ Aquatic Sensor Network Technology, Storrs, CT, 06268
}

\begin{abstract}
In this paper, we consider a problem of localizing a moving object in the context of an underwater distributed antenna system. All the distributed nodes record the time-of-arrivals of a single message from the moving object and obtain the Doppler speed estimates used in the decoding process. Conventional methods have relied only on the time-of-arrival measurements to obtain position estimates, on top of which filtering methods can be further applied for tracking purposes. In this work, we explore the usefulness of Doppler speed information. The combination of time-of-arrival measurements and Doppler speed estimates associated with a single message enhances the accuracy of position estimation and provides an estimate of the velocity of the mobile object. A Kalman Filter (KF) and a Probabilistic Data Association Filter (PDAF) are further implemented processing the point estimates. Simulations show that the proposed Doppler-aided methods improve both the point estimation and tracking filter performance, which is verified using data from pool tests.

Keywords: Underwater acoustic localization, distributed antenna systems, Doppler estimation, tracking
\end{abstract}

\footnotetext{
${ }^{*}$ Corresponding Author: Shengli Zhou, Department of Electrical and Computer Engineering, 371 Fairfield Way Unit-4157, University of Connecticut, Storrs, CT 06269-4157, USA; Email: shengli@engr.uconn.edu; Phone: 1-860-486-4593.
} 


\section{Introduction}

In underwater acoustic communications there has seen a growing interest in one system architecture: Distributed Antenna Systems (DAS). This differs from the common Central Antenna System (CAS) in that multiple antennas are distributed throughout a wireless network and connected via some outside links (either cabling or in the case of underwater acoustic networks a surface link connecting nodes via radio). The advantages of DAS in terms of throughput, outage performance, coverage area, and other properties are well discussed elsewhere $[1,2,3,4,5]$. What is of note for this particular topic is the notion of fusing data from multiple antennas in the network to enable near-instantaneous location estimation for highly mobile network elements, such as fast-moving AUV's, gliders, or other non-tethered nodes in relatively sparse networks.

Localization is a key part of any wireless network or vehicle navigation scheme, and is a particularly challenging task in the realm of underwater acoustic communications. Much work has been done with regards to underwater localization in recent years, as summarized in $[6,7]$. Most works focus on using ranging or angle estimates as the primary methods of location estimation, and perform multilateration using information from multiple anchor nodes $[8,9,10$, $11,12,13,14,15]$. Other methods have been developed from a network perspective, working largely to extend the multilateration technique to cover large areas or exploit the broadcast nature of wireless transmissions to reduce the communication overhead $[16,17,18]$. In our previous work, we had developed a system that used scheduled periodic broadcasts from timesynchronized anchors [19] and another system with asynchronous anchors that can react to a localization demand [20].

When several messages are required for time-of-flight multilateration or similar methods, there arises an important issue when localizing mobile elements. The nature of the underwater acoustic channel forces these messages to have a large temporal delay. Consequently, as the mobile elements are moving, there would be non-negligible position changes in the data collection process. This typically must be dealt with in the estimation itself, either by using non-linear 
tracking or measurement conversion methods or supplementing with measurements from inertial navigation systems (INS).

In this work, we consider the localization of a mobile unit via a distributed antenna system which only requires one message to be sent from the mobile unit. Note that Doppler speed estimation is used often as a tool to improve messaging capabilities in underwater communications. The modems used in our research have the capacity to estimate the channel during receiver processing and also provide an estimate of the Doppler scaling factor present in the received signal $[21,22]$. With a received message, all the distributed nodes in a DAS record the time-of-arrivals and obtain the Doppler speed estimates used in the decoding process. Conventional methods have relied only on the time-of-arrival measurements to obtain position estimates, on top of which filtering methods can be further applied for tracking purposes. In this paper, we explore the usefulness of Doppler speed information. The combination of time-of-arrival measurements and Doppler speed estimates associated with a single message enhances the accuracy of position estimation and provides an estimate of the velocity of the mobile object. A Kalman Filter (KF) and a Probabilistic Data Association Filter (PDAF) are then further implemented processing the point estimates.

This Doppler estimation along with an estimation of the time-of-flight for a message from a target node to several anchor nodes constitutes what can be considered a range and range-rate measurement. The use of range-rate to supplement range measurements has been studied in the radar and sonar literature, and generally it would appear that it can affect the localization and tracking accuracy both positively and negatively. The primary factor in whether the effect is an improvement in accuracy or a reduction is based on the uncertainty of the range-rate estimate. We look at experimental data where the Doppler speed estimation capability of a modem is analyzed, and determine if the uncertainty is low enough to offer performance improvement.

The primary contribution of this paper is to derive an instantaneous position and velocity estimator for a mobile node in a distributed antenna system based on both the time-of-arrival 
measurements and the Doppler speed estimates, perform simulated analysis to determine the performance improvement due to the Doppler information, and then validate the assumptions and simulations with tests using acoustic modems that enable this capability.

The paper is organized as follows. Section 2 describes the general protocol and algorithm to determine the position of a moving unit. Section 3 describes the trackers adopted. Sections 4 and 5 present the simulation and test results, respectively. Section 6 features discussions of alternative methods and scenario-specific concerns, and finally we conclude our work in Section 7.

Notation: Bold upper case and lower case letters denote matrices and column vectors, respectively; $(\cdot)^{T}$ denotes transpose of a vector or a matrix.

\section{Localization Protocol}

We consider a scenario of AUV navigation, wherein we have one cooperative target attempting to navigate with the aid of several listening beacons deployed over an area of interest. An AUV, denoted as the active node $\mathrm{s}$, is located at an arbitrary position $\left[x_{\mathrm{s}}, y_{\mathrm{s}}, z_{\mathrm{s}}\right]$ within a known area. We assume that $N$ antenna nodes are deployed at positions $\left[x_{n}, y_{n}, z_{n}\right], n=1,2, \ldots, N$.

There are several assumptions we must explicitly make with regards to the node properties. First, the active antenna nodes can all communicate perfectly in the respective frames of localization and there are no interference nor collisions (discussion on communications failure is deferred to Section 6). Second, all antenna nodes have perfect knowledge of their position and they share a globally synchronized clock. Third, the antennas are capable of collaboratively sharing information such that one arbitrary node can collect all measurements before either localizing the active node at the antenna and relaying that to the node, or transmitting the collected information back to the active node for it to self-localize. This can either be handled through messaging among antenna nodes, or more elegantly via DAS networking with a secondary communication link. 


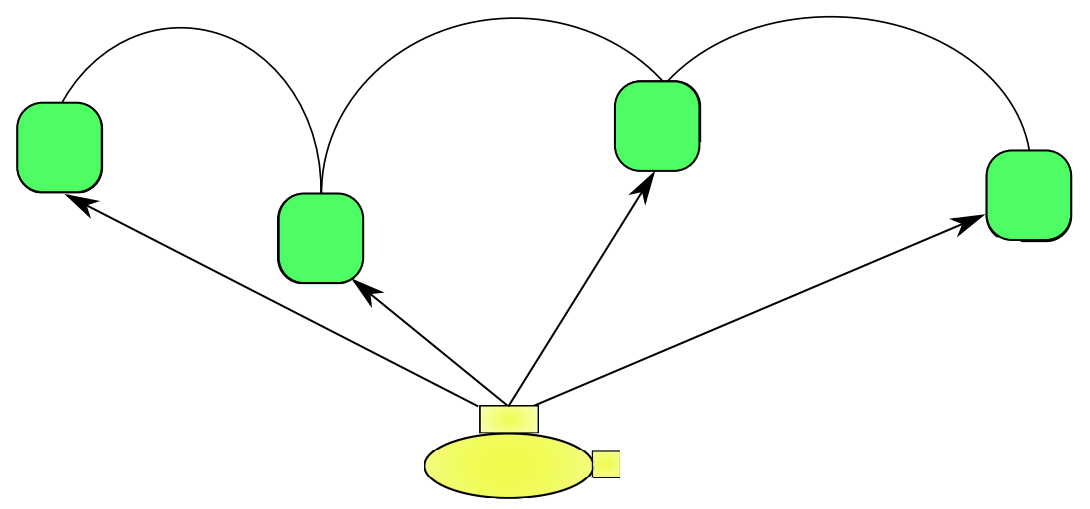

Figure 1: A brief overview of the transmission protocol in a sample network. The AUV sends a burst message received by all nearby antenna nodes, which then collaborate among each other to localize the AUV.

The procedure would then be as follows:

- At some time $t_{0}$, the active node transmits a single message requesting localization be performed by all listening nodes, as well as the local time $t_{0}$ at which the message was transmitted. Each node $n$ then receives that message at local time $\hat{t}_{n}$.

- Each node obtains a Doppler speed estimate $\hat{v}_{n}$ based on the received waveform.

- After collecting all information, a master node obtains all estimates and performs the localization procedure and transmits the full state estimate back to the active node. Alternatively, the information could be collected and relayed to the active node where localization is then performed.

- As point estimates are collected, tracking algorithms can be used to track the active node.

\subsection{Measurements and Estimation}

Since we have assumed that there are only two effective clocks in the system, the active clock and the shared antenna clock, we can assume that the antenna clock is a reference and from there we only need to consider the separate active node clock. 
At an antenna node $n$, for a given time period $k$, the true message propagation delay from the active node $\mathrm{s}$ is:

$$
t_{\mathrm{s}, n}(k)=\frac{1}{c} d_{\mathrm{s}, n}(k),
$$

where $c$ is the assumed (fixed) propagation speed of sound in water and $d_{\mathrm{s}, n}(k)$ is the distance between the active node and antenna node $n$ defined as:

$$
d_{\mathrm{s}, n}(k)=\sqrt{\left(x_{\mathrm{s}}(k)-x_{n}\right)^{2}+\left(y_{\mathrm{s}}(k)-y_{n}\right)^{2}+\left(z_{\mathrm{s}}(k)-z_{n}\right)^{2}} .
$$

Our estimated time of arrival is then given as:

$$
\hat{t}_{\mathrm{s}, n}(k)=\hat{t}_{n}-t_{0}=\frac{1}{c} d_{\mathrm{s}, n}(k)+O_{\mathrm{s}}(k)+w_{\mathrm{t}, n}(k),
$$

where $O_{\mathrm{s}}(k)$ is the active node clock offset at time $k$ and $w_{\mathrm{t}, n}(k)$ is the measurement noise at node $n$ for time $k$.

The Doppler speed measured at each node is the absolute speed difference between the active node and the antenna node modified by the three-dimensional angle between the two nodes. Assuming the antenna nodes are not moving, the magnitude of a velocity is given as:

$$
\left|v_{n}(k)\right|=\sqrt{\dot{x}_{\mathrm{s}}(k)^{2}+\dot{y}_{\mathrm{s}}(k)^{2}+\dot{z}_{\mathrm{s}}(k)^{2}} .
$$

The total angle between an antenna and the active node is denoted as the sum of the $x-y$ angle $(\cos (\alpha))$ and the $z$-plane $(\sin (\gamma))$ given respectively as:

$$
\cos (\alpha(k))=\frac{x_{\mathrm{s}}(k)-x_{n}}{d_{\mathrm{s}, n}(k)}+\frac{y_{\mathrm{s}}(k)-y_{n}}{d_{\mathrm{s}, n}(k)}
$$




$$
\sin (\gamma(k))=\frac{z_{\mathrm{s}}(k)-z_{n}}{d_{\mathrm{s}, n}(k)}
$$

The Doppler speed is then given as:

$$
\begin{aligned}
v_{n}(k) & =\left|v_{n}(k)\right|(\cos (\alpha(k))+\sin (\gamma(k))) \\
& =\sqrt{\dot{x}_{\mathrm{s}}(k)^{2}+\dot{y}_{\mathrm{s}}(k)^{2}+\dot{z}_{\mathrm{s}}(k)^{2}}\left(\frac{x_{\mathrm{s}}(k)-x_{n}}{d_{\mathrm{s}, n}(k)}+\frac{y_{\mathrm{s}}(k)-y_{n}}{d_{\mathrm{s}, n}(k)}+\frac{z_{\mathrm{s}}(k)-z_{n}}{d_{\mathrm{s}, n}(k)}\right) .
\end{aligned}
$$

Since we assume that all four antenna nodes are traveling at zero velocity, the only difference for the various estimates is in the relative positioning of the nodes, which is a function of their known locations and the singular unknown location of the active node.

The modems used in our experiments directly estimate this value of $v_{n}(k)$ without the need for further alteration, and we can represent the estimate as:

$$
\hat{v}_{n}(k)=v_{n}(k)+w_{\mathrm{v}, n}(k),
$$

where $w_{\mathrm{v}, n}(k)$ is the Doppler estimation error for time $k$ at node $n$.

Based on the previous assumptions, for a given time instant $k$, a localization transmission will have generated a set of $2 N$ measurements, where $N$ is the total number of participating antenna nodes. Given this, we can form the maximum-likelihood (ML) multilateration solution for the active node position as well as the likelihood estimate for the velocity and combine them to form a joint likelihood of the full 6-dimensional state of the active node at time instant $k$.

\subsection{Position Estimation based on Time-of-Arrivals}

The position at time $k$ is defined as a three-dimensional vector as

$$
\ell(k)=\left[x_{\mathrm{s}}(k), y_{\mathrm{s}}(k), z_{\mathrm{s}}(k)\right] .
$$


Due to the clock difference between the mobile unit and the antenna nodes, time differencing is applied to obtain time-difference of arrival (TDOA) estimates as

$$
\Delta \hat{t}_{n, 1}(k)=\hat{t}_{\mathrm{s}, n}(k)-\hat{t}_{\mathrm{s}, 1}(k), \quad n=2, \ldots, N
$$

In the presence of clock bias and noise, one has:

$$
\begin{aligned}
\Delta \hat{t}_{n, 1}(k) & =\frac{1}{c} d_{\mathrm{s}, n}(k)+O_{\mathrm{s}}(k)+w_{\mathrm{t}, n}(k)-\left[\frac{1}{c} d_{\mathrm{s}, 1}(k)+O_{\mathrm{s}}(k)+w_{\mathrm{t}, 1}(k)\right] \\
& =\frac{1}{c}\left[d_{\mathrm{s}, n}(k)-d_{\mathrm{s}, 1}(k)\right]+w_{\mathrm{t}, n}(k)-w_{\mathrm{t}, 1}(k) .
\end{aligned}
$$

With the timing offset eliminated, and assuming that the noises are zero-mean additive Gaussian, the ML estimator for the position would be (similar to [19, 23]):

$$
\hat{\boldsymbol{\ell}}(k)=\underset{\boldsymbol{\ell}(k)}{\arg \min } \frac{1}{2\left(c \sigma_{\mathrm{t}}\right)^{2}} \sum_{n=2}^{N}\left[c \Delta \hat{t}_{n, 1}(k)-\left(d_{\mathrm{s}, n}(k)-d_{\mathrm{s}, 1}(k)\right)\right]^{2},
$$

where $\sigma_{\mathrm{t}}$ is the standard deviation on the time-of-arrival estimation error.

Alternatively, we can note that the TDOA estimates are correlated by subtracting the first measurement from other measurements. This correlation can be corrected for by denoting an $(N-1) \times(N-1)$ covariance matrix:

$$
\mathbf{P}=\left[\begin{array}{cccc}
1 & 0.5 & \ldots & 0.5 \\
0.5 & 1 & \ddots & 0.5 \\
\vdots & \ddots & \ddots & \vdots \\
0.5 & \ldots & 0.5 & 1
\end{array}\right]
$$


Modifying (13), we have

$$
\hat{\boldsymbol{\ell}}(k)=\underset{\boldsymbol{\ell}(k)}{\arg \min }\left[c \Delta \mathbf{t}_{n, 1}(k)-\mathbf{d}_{\mathrm{s}, n}(k)+d_{\mathrm{s}, 1}(k) \mathbf{1}\right] \mathbf{P}^{-1}\left[c \Delta \mathbf{t}_{n, 1}(k)-\mathbf{d}_{\mathrm{s}, n}(k)+d_{\mathrm{s}, 1}(k) \mathbf{1}\right]
$$

where $\Delta \mathbf{t}_{n, 1}(k)$ and $\mathbf{d}_{\mathrm{s}, n}(k)$ are $(N-1) \times 1$ vectors collecting $N-1$ relevant scalar values, and $\mathbf{1}$ is a $(N-1) \times 1$ identity vector. The method in (15) is termed as "position-ML" in numerical results.

\subsection{Position and Velocity Estimation based on Time-of-Arrivals and Doppler Speed Estimates}

Note that the Doppler speed measurement depends on both on the speed of the active node as well as the position of the active node relative to the antenna nodes (as this affects the angle of incidence and thus the Doppler speed). We define a state vector:

$$
\boldsymbol{\theta}(k)=\left[x_{\mathrm{s}}(k), \dot{x}_{\mathrm{s}}(k), y_{\mathrm{s}}(k), \dot{y}_{\mathrm{s}}(k), z_{\mathrm{s}}(k), \dot{z}_{\mathrm{s}}(k)\right],
$$

where $\left({ }^{*}\right)$ denotes the first-order derivative.

Again assuming zero-mean additive Gaussian noise the joint likelihood for the active node $s$ is:

$$
\hat{\boldsymbol{\theta}}(k)=\underset{\boldsymbol{\theta}(k)}{\arg \min } \frac{1}{2\left(c \sigma_{\mathrm{t}}\right)^{2}} \sum_{n=2}^{N}\left[c \Delta \hat{t}_{n, 1}(k)-\left(d_{\mathrm{s}, n}(k)-d_{\mathrm{s}, 1}(k)\right)\right]^{2}+\frac{1}{\sigma_{\mathrm{v}}^{2}} \sum_{n=1}^{N}\left[\hat{v}_{n}(k)-v_{n}(k)\right]^{2},
$$

where

$$
v_{n}(k)=\sqrt{\dot{x}_{\mathrm{s}}(k)^{2}+\dot{y}_{\mathrm{s}}(k)^{2}+\dot{z}_{\mathrm{s}}(k)^{2}}\left(\frac{x_{\mathrm{s}}(k)-x_{n}}{d_{\mathrm{s}, n}(k)}+\frac{y_{\mathrm{s}}(k)-y_{n}}{d_{\mathrm{s}, n}(k)}+\frac{z_{\mathrm{s}}(k)-z_{n}}{d_{\mathrm{s}, n}(k)}\right),
$$

and $\sigma_{\mathrm{v}}$ is the standard deviation on the Doppler speed estimation error (estimated by the receiving modem or assumed estimated for the deployment). We solve this maximum likelihood

problem by first using a coarse search grid to find an initial point, after which we perform a 
gradient-descent search to find the optimal solution. This method is termed as "Doppler-ML" in numerical results.

\section{Tracking}

Since this form of localization implies a mobile object that requires instantaneous point estimation, a logical extension would be to supplement positioning with tracking algorithms. To this end, we first consider simple models, namely the Kalman Filter (KF) and Probabilistic Data Association Filter (PDAF). Note that the trackers are applied to the estimates of $\boldsymbol{\theta}$, not to the measurements directly; this implicitly assumes that our estimates are linear. In Section 6 we briefly discuss the validity of a linear motion assumption and the scenarios in which it would likely be applicable. The development in this section follows standard texts [24, 25].

\subsection{Kalman Filtering based on Position Estimates}

Similar to our previous work in [19], we model the movement of the node on each direction with a white noise acceleration (DWNA) model, with each direction acting as a separate uncoupled process; that is, $x, y$ and $z$. Further, in order to gauge the relative accuracy of the velocity estimates, we can use a Kalman Filter (KF) on the position-only estimates to obtain a velocity estimate and compare them to the Doppler based position and velocity estimates.

For the position-only estimates there are three-dimensional state (position) vectors and a KF would then estimate the six-dimension state (position and velocity). This is termed as "positionKF" in the numerical results. The state variable is $\boldsymbol{\theta}(k)$ as defined in (16). The state-space equations are as follows.

$$
\boldsymbol{\theta}(k+1)=\mathbf{F} \boldsymbol{\theta}(k)+\boldsymbol{\zeta}(k)
$$


with measurement

$$
\mathbf{z}(k+1)=\mathbf{H} \boldsymbol{\theta}(k+1)+\mathbf{w}(k+1)
$$

where

$$
\begin{aligned}
\mathbf{F} & =\left[\begin{array}{llllll}
1 & \tau & 0 & 0 & 0 & 0 \\
0 & 1 & 0 & 0 & 0 & 0 \\
0 & 0 & 1 & \tau & 0 & 0 \\
0 & 0 & 0 & 1 & 0 & 0 \\
0 & 0 & 0 & 0 & 1 & \tau \\
0 & 0 & 0 & 0 & 0 & 1
\end{array}\right], \\
\mathbf{H} & =\left[\begin{array}{llllll}
1 & 0 & 0 & 0 & 0 & 0 \\
0 & 0 & 1 & 0 & 0 & 0 \\
0 & 0 & 0 & 0 & 1 & 0
\end{array}\right],
\end{aligned}
$$

$\boldsymbol{\zeta}(k)$ is the process noise, $\mathbf{w}(k)$ is measurement noise and $\tau$ is the sampling interval of the discrete model in seconds. The state covariance is modeled as

$$
\mathbf{P}(k+1 \mid k)=\mathbf{F P}(k \mid k) \mathbf{F}^{T}+\mathbf{Q},
$$


where the process noise has a covariance matrix as $[24,25]$

$$
\mathbf{Q}=\left[\begin{array}{cccccc}
\frac{1}{4} \tau^{4} & \frac{1}{2} \tau^{3} & 0 & 0 & 0 & 0 \\
\frac{1}{2} \tau^{3} & \tau^{2} & 0 & 0 & 0 & 0 \\
0 & 0 & \frac{1}{4} \tau^{4} & \frac{1}{2} \tau^{3} & 0 & 0 \\
0 & 0 & \frac{1}{2} \tau^{3} & \tau^{2} & 0 & 0 \\
0 & 0 & 0 & 0 & \frac{1}{4} \tau^{4} & \frac{1}{2} \tau^{3} \\
0 & 0 & 0 & 0 & \frac{1}{2} \tau^{3} & \tau^{2}
\end{array}\right] \sigma_{\mathrm{q}}^{2}
$$

Here, $\sigma_{\mathrm{q}}$ is a design parameter that is chosen to match the most likely level of process noise to be experienced by the object in question; which is to say it controls how much the model anticipates the object to maneuver. Given that the object in question is likely to have its velocity at a slow, steady rate, a process noise level of $\sigma_{\mathrm{q}}=0.1 \mathrm{~m} / \mathrm{s}^{2}$ was selected to best emulate this behavior. The filter was initialized with a two-point initialization.

\subsection{Kalman Filtering based on Position and Velocity Estimates}

The Doppler-aided estimates are six-dimensional state (position and velocity) vectors and are given to the KF to provide estimates of the nine-dimension state (position, velocity, and acceleration). We chose to use a higher-order filter for the Doppler-aided estimates as when we simulated both the second and third order filters, the higher-order filter provided a nonnegligible improvement in root-mean-square error (RMSE). Thus, the applicable higher order filter was determined to be worth the increased computation [24]. The resulting filter is termed as "Doppler-KF" in the numerical results. With a new state vector defined as

$$
\tilde{\boldsymbol{\theta}}(k)=[x(k), \dot{x}(k), \ddot{x}(k), y(k), \dot{y}(k), \ddot{y}(k), z(k), \dot{z}(k), \ddot{z}(k)],
$$


the state-space model is

$$
\tilde{\boldsymbol{\theta}}(k+1)=\tilde{\mathbf{F}} \tilde{\boldsymbol{\theta}}(k)+\tilde{\boldsymbol{\zeta}}(k)
$$

with measurement

$$
\tilde{\mathbf{z}}(k+1)=\tilde{\mathbf{H}} \Phi(k+1)+\tilde{\mathbf{w}}(k+1)
$$

where

$$
\tilde{\mathbf{F}}=\left[\begin{array}{ccccccccc}
1 & \tau & \tau^{2} & 0 & 0 & 0 & 0 & 0 & 0 \\
0 & 1 & \tau & 0 & 0 & 0 & 0 & 0 & 0 \\
0 & 0 & 1 & 0 & 0 & 0 & 0 & 0 & 0 \\
0 & 0 & 0 & 1 & \tau & \tau^{2} & 0 & 0 & 0 \\
0 & 0 & 0 & 0 & 1 & \tau & 0 & 0 & 0 \\
0 & 0 & 0 & 0 & 0 & 1 & 0 & 0 & 0 \\
0 & 0 & 0 & 0 & 0 & 0 & 1 & \tau & \tau^{2} \\
0 & 0 & 0 & 0 & 0 & 0 & 0 & 1 & \tau \\
0 & 0 & 0 & 0 & 0 & 0 & 0 & 0 & 1
\end{array}\right],
$$

$$
\tilde{\mathbf{H}}=\left[\begin{array}{lllllllll}
1 & 0 & 0 & 0 & 0 & 0 & 0 & 0 & 0 \\
0 & 1 & 0 & 0 & 0 & 0 & 0 & 0 & 0 \\
0 & 0 & 0 & 1 & 0 & 0 & 0 & 0 & 0 \\
0 & 0 & 0 & 0 & 1 & 0 & 0 & 0 & 0 \\
0 & 0 & 0 & 0 & 0 & 0 & 1 & 0 & 0 \\
0 & 0 & 0 & 0 & 0 & 0 & 0 & 1 & 0
\end{array}\right] .
$$


The state covariance is updated as

$$
\tilde{\mathbf{P}}(k+1 \mid k)=\tilde{\mathbf{F}} \tilde{\mathbf{P}}(k \mid k) \tilde{\mathbf{F}}^{T}+\tilde{\mathbf{Q}},
$$

where the process noise has a covariance matrix $[24,25]$

$$
\tilde{\mathbf{Q}}=\left[\begin{array}{ccccccccc}
\frac{1}{4} \tau^{4} & \frac{1}{2} \tau^{3} & \frac{1}{2} \tau^{2} & 0 & 0 & 0 & 0 & 0 & 0 \\
\frac{1}{2} \tau^{3} & \tau^{2} & \tau & 0 & 0 & 0 & 0 & 0 & 0 \\
\frac{1}{2} \tau^{2} & \tau & 1 & 0 & 0 & 0 & 0 & 0 & 0 \\
0 & 0 & 0 & \frac{1}{4} \tau^{4} & \frac{1}{2} \tau^{3} & \frac{1}{2} \tau^{2} & 0 & 0 & 0 \\
0 & 0 & 0 & \frac{1}{2} \tau^{3} & \tau^{2} & \tau & 0 & 0 & 0 \\
0 & 0 & 0 & \frac{1}{2} \tau^{2} & \tau & 1 & 0 & 0 & 0 \\
0 & 0 & 0 & 0 & 0 & 0 & \frac{1}{4} \tau^{4} & \frac{1}{2} \tau^{3} & \frac{1}{2} \tau^{2} \\
0 & 0 & 0 & 0 & 0 & 0 & \frac{1}{2} \tau^{3} & \tau^{2} & \\
0 & 0 & 0 & 0 & 0 & 0 & \frac{1}{2} \tau^{2} & \tau & 1
\end{array}\right] \sigma_{\mathrm{q}}^{2}
$$

Again, the straight line motion warrants a low process noise, and $\sigma_{\mathrm{q}}=0.5 \mathrm{~m} / \mathrm{s}^{2}$ was selected. The filter was initialized with a two-point initialization [24].

\subsection{Probabilistic Data Association Filter}

Previously in [19] we had found that a PDAF could offer better performance to a KF in our particular scenario if there was some non-zero probability that a transmission could be successfully received and decoded but have a very large amount of error in the time-of-arrival estimate at the antenna node. In that context, the PDAF offers an improved performance over the standard KF, by allowing outlier estimates such as those to be ignored as false-alarm or clutter detections [25].

The PDAF is very similar to the KF in terms of state equations. Here we use position-KF as an example, but Doppler-KF can be modified in the same fashion. The state-space equation 
is

$$
\boldsymbol{\theta}(k+1)=\mathbf{F} \boldsymbol{\theta}(k)+\boldsymbol{\zeta}(k)
$$

with measurement

$$
\mathbf{z}(k+1)=\mathbf{H} \boldsymbol{\theta}(k+1)+\mathbf{w}(k+1)
$$

where $\mathbf{F}$ and $\mathbf{H}$ are given in (21) and (22). The state covariance matrix is updated as before

$$
\mathbf{P}(k+1 \mid k)=\mathbf{F P}(k \mid k) \mathbf{F}^{T}+\mathbf{Q} .
$$

The difference is on how to compute $\mathbf{P}(k \mid k)$, detailed as follows.

Define

$$
\begin{aligned}
\boldsymbol{\nu}(k) & =\mathbf{z}(k)-\mathbf{H} \boldsymbol{\theta}(k) \\
\mathbf{S}(k) & =\mathbf{H P}(k \mid k-1) \mathbf{H}^{T}+\mathbf{R} \\
\mathbf{W}(k) & =\mathbf{P}(k \mid k-1) \mathbf{H}^{T} \mathbf{S}^{-1}(k) \\
e(k) & =e^{-\frac{1}{2} \nu(k)^{T} \mathbf{S}(k)^{-1} \nu(k)}
\end{aligned}
$$

where $\mathbf{R}$ is the covariance matrix for the observation noise. Let $P_{\mathrm{D}}$ denote the probability of detection, which is a design parameter. Assume that the measurement is always gated, and that there is only a single target and a single measurement at each time step. Accordingly, $P_{\mathrm{D}}$ is the probability that the current measurement is a valid estimate (that is to say, an estimate that is consistent with the state estimate and state covariance of the filter) of the node being tracked. 
With

$$
b=2\left(\frac{1-P_{\mathrm{D}}}{P_{\mathrm{D}}}\right)
$$

the probability of no correct measurement available (meaning that the measurement provided is so corrupted as to be "false") is

$$
\beta_{0}(k)=\frac{b}{b+e(k)}
$$

and the probability of a correct measurement is

$$
\beta_{1}(k)=\frac{e(k)}{b+e(k)}
$$

With

$$
\begin{aligned}
\mathbf{P}_{1}(k \mid k) & =\mathbf{P}(k \mid k-1)-\mathbf{W}(k) \mathbf{S}(k) \mathbf{W}(k)^{T}, \\
\mathbf{P}_{2}(k) & =\mathbf{W}(k)\left(\beta_{1}(k) \boldsymbol{\nu}(k) \boldsymbol{\nu}(k)^{T}-\beta_{1}^{2}(k) \boldsymbol{\nu}(k) \boldsymbol{\nu}(k)^{T}\right) \mathbf{W}(k)^{T},
\end{aligned}
$$

the covariance matrix is updated as

$$
\mathbf{P}(k \mid k)=\beta_{0}(k) \mathbf{P}(k \mid k-1)+\beta_{1}(k) \mathbf{P}_{1}(k \mid k)+\mathbf{P}_{2}(k) .
$$

Based on experiments in [19], the number of "false detection" measurements was around 5-10\% of the total samples, and so a value of 0.95 was selected for $P_{\mathrm{D}}$ in our numerical results.

\section{Simulations}

A simple scenario was used where a mobile unit with a straight line motion passes through a grid of antenna nodes, with a varying message update frequency. To provide context for the 


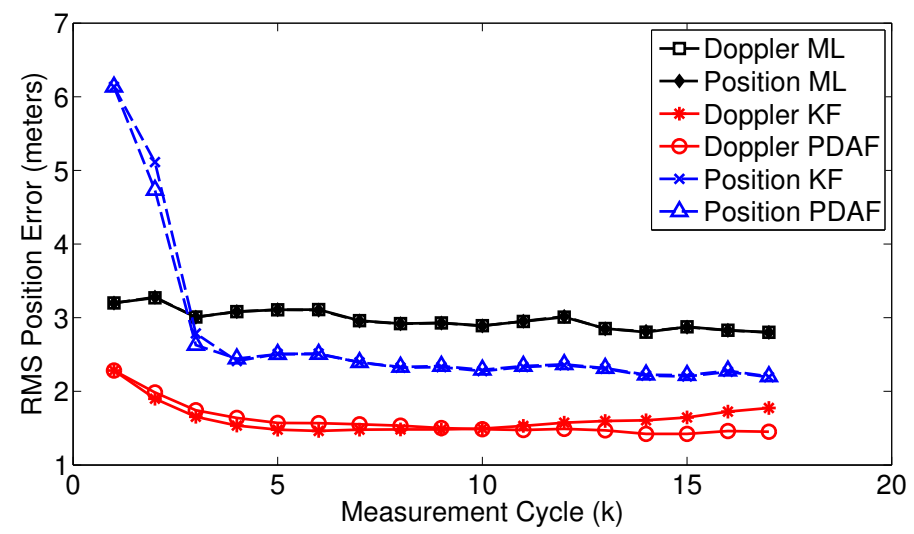

Figure 2: Comparison of simulated position error for the measurement, KF, and PDAF for a transmission interval of $5 \mathrm{~s}$.

effects of the Doppler speed estimate, we will compare the results with the tracking algorithms similar to what we developed in [19]. All filter parameters (namely process noise) are the same where applicable to provide a more valid comparison.

Further, the measurement noises used in simulation were drawn from experiments that determined the accuracy of both the time-of-arrival estimates [20] and the Doppler speed estimates. In the latter case, a single transmitter was moved in an athletic pool for approximately 100 transmissions at a fixed velocity, and the accuracy was estimated from the sample variance.

We consider a grid of four anchor nodes located on the same $z$-plane defined as $z=0$ arranged in a square separated by $300 \mathrm{~m}$. Our active node begins positioned in this grid at $(x, y, z)=(75,25,10)$ in meters and has a constant velocity defined as $(\dot{x}, \dot{y}, \dot{z})=(-.5, .7, .4)$ in meters/second. There are 20 total measurements during the scenario and we perform 1000 Monte-Carlo runs for each scenario. The simulated noise level is $c \sigma_{\mathrm{t}}=0.825 \mathrm{~m}$ and $\sigma_{\mathrm{v}}=$ $0.07 \mathrm{~m} / \mathrm{s}$, where $w_{\mathrm{t}, n} \sim \mathcal{N}\left(0, \sigma_{\mathrm{t}}^{2}\right)$ and $w_{\mathrm{v}, n} \sim \mathcal{N}\left(0, \sigma_{\mathrm{v}}^{2}\right)$.

In the first scenario, we consider a message update interval of $5 \mathrm{~s}$. The position and velocity errors are given in Fig. 2 and Fig. 3, respectively. Both of the tracking filters in this case assumed a process noise of $\sigma_{\mathrm{q}}=0.5 \mathrm{~m} / \mathrm{s}^{2}$, representative of a very small amount of velocity change. For the filters with the Doppler-aided estimates, the PDAF is out-performed by the KF 


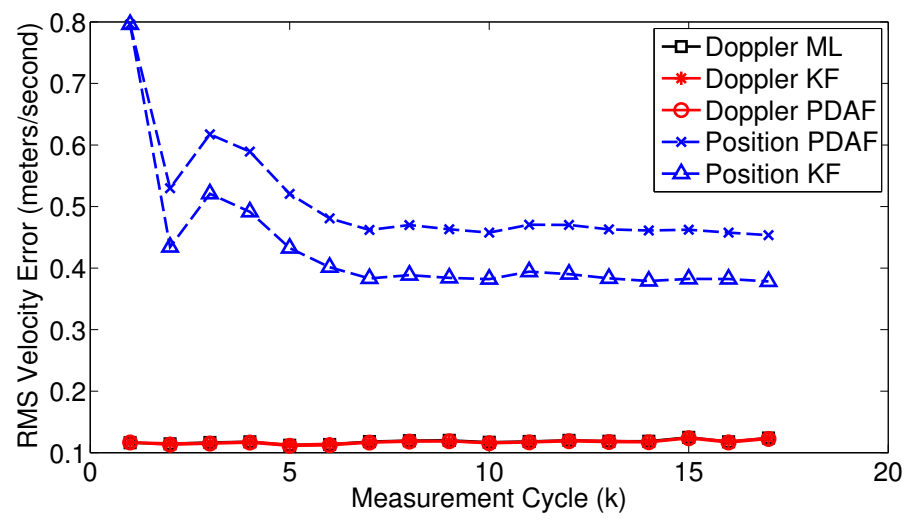

Figure 3: Comparison of simulated velocity error for the measurement, KF, and PDAF for a transmission interval of $5 \mathrm{~s}$.

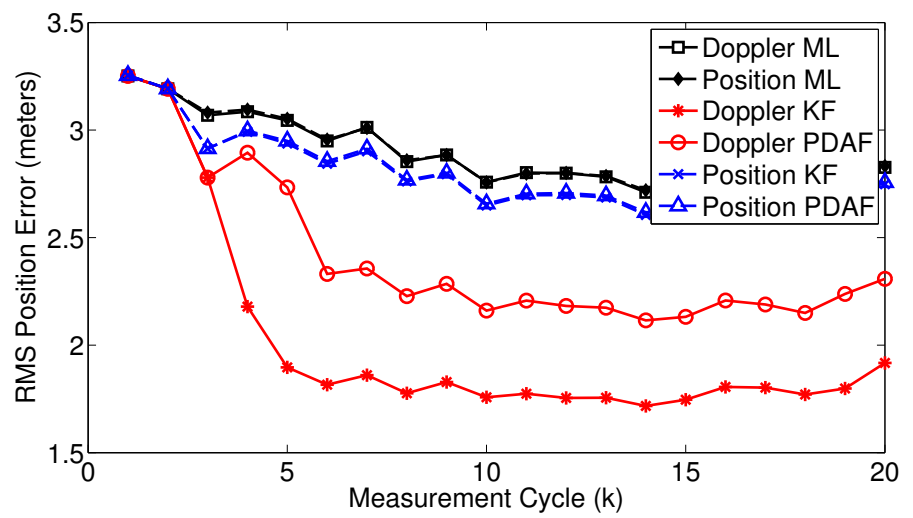

Figure 4: Comparison of simulated position error for the measurement, KF, and PDAF for a transmission interval of $10 \mathrm{~s}$.

in terms of root-mean-square (RMS) position error. This gap is due to the lack of simulated false detections; as the probability of detection is increased in the PDAF it becomes identical to the Kalman Filter. However, both are improvements over the raw position error and also are far better then their position-only counterparts. In the velocity error, the Doppler included estimators perform almost identically and have error almost exactly the same as the raw estimated velocity. The position-only counterparts are much worse in comparison.

The second scenario is similar to the first, with the same noise assumptions and node distributions. However, the message update interval is $10 \mathrm{~s}$. There are still 20 transmissions in 


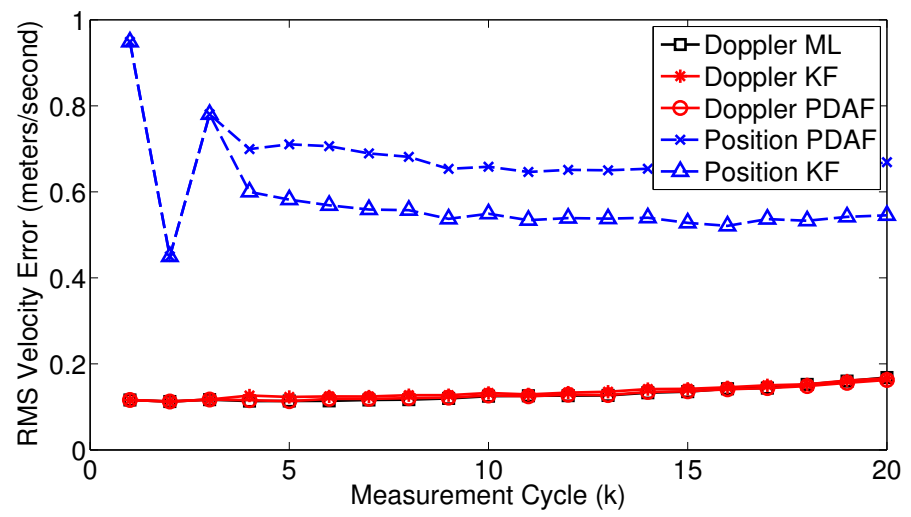

Figure 5: Comparison of simulated velocity error for the measurement, KF, and PDAF for a transmission interval of $10 \mathrm{~s}$.

total, and the node starts at the same point and travels with the same speed, which means that there is a greater distance traveled between transmissions and overall. The position and velocity errors are given in Fig. 4 and Fig. 5, respectively. As can be expected, the general level of error increased for all estimators; however, the general performance gaps appear to be approximately the same. The exception is in the case of the widening gap of the velocity estimates, as the purely tracked estimates are performing poorer compared to the Doppler-aided estimators.

For another set of scenarios, we considered altering the value of $\sigma_{q}$. Fig. 6 and Fig. 7 show the position and velocity for a value of $\sigma_{q}=0.01$, Fig. 8 and Fig. 9 show the position and velocity for a value of $\sigma_{q}=0.1$, Fig. 10 and Fig. 11 show the position and velocity for a value of $\sigma_{q}=1$. As the value of $\sigma_{q}$ is altered, the position only performance varies drastically, with a visible trade-off between initial and asymptotic accuracy, while the Doppler-aided have a much smaller variation in performance and consistently provide superior accuracy in either position or velocity estimate or both.

\section{Pool Testing}

We performed testing of the algorithm at the UCONN Brundage Pool on June 24, 2014. For the test, five AquaSeNT Modems were utilized to act as both mobile and antenna nodes. Four 


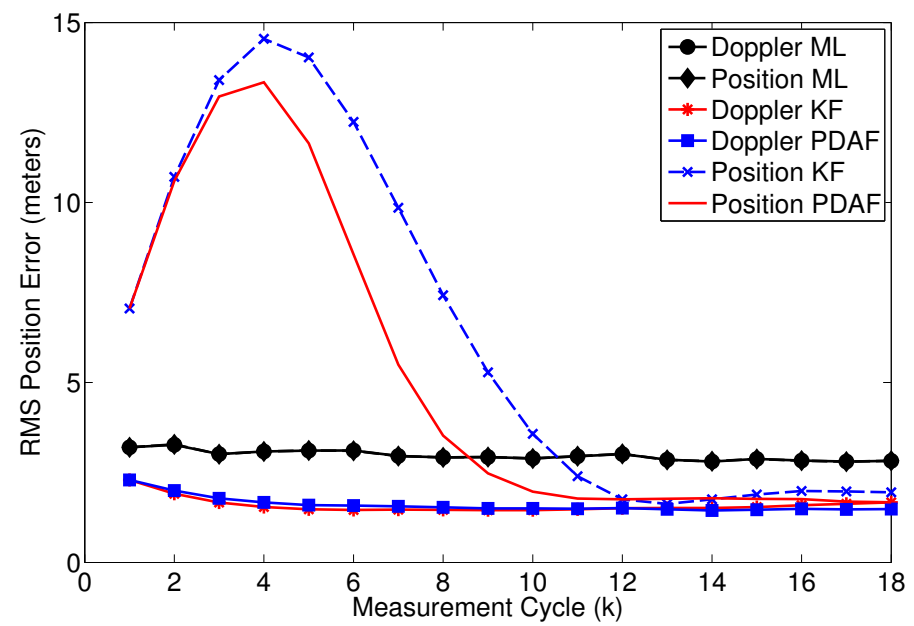

Figure 6: Comparison of simulated position error for the measurement, KF, and PDAF with $\sigma_{q}=0.01$.

of the nodes were deployed to the approximate corners of the pool, and the fifth was repeatedly moved along the long edge in the center lane at a near-constant velocity. A layout of nodes in the pool with the appropriate dimensions is presented in Fig. 12. Each of the modems had the transducer array submerged approximately 1 meter below the surface.

During the movement, the modem transmitted a beaconing message every 4 seconds. Processing of the received messages was performed offline. Four test runs were performed: the first was 24 messages long and had an average velocity of $0.24 \mathrm{~m} / \mathrm{s}$, the second 23 messages long and had an average velocity of $0.25 \mathrm{~m} / \mathrm{s}$, the third 22 messages long and had an average velocity of $0.26 \mathrm{~m} / \mathrm{s}$, and the fourth 26 messages long and had an average velocity of $0.22 \mathrm{~m} / \mathrm{s}$. The position and velocity errors for the estimates of each set are in Fig. $13 \& 14,15 \& 16,17 \& 18$, and 19 $\& 20$, respectively.

One important thing to note about the pool tests is that while it was attempted to use four antenna nodes, one of the nodes experienced consistent communication failures over the course of all four runs. Therefore, the estimates presented are calculated using only three antenna nodes, and the estimators are supplied the true depth of the moving transmitter to perform their estimation (though this is not entirely inconsistent with a real scenario, as depth is easily 


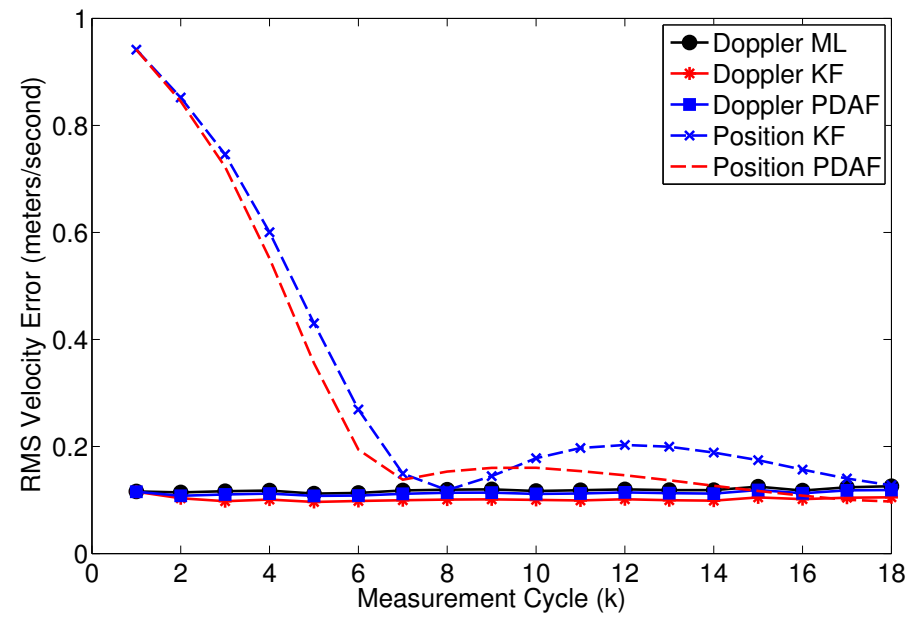

Figure 7: Comparison of simulated velocity error for the measurement, KF, and PDAF with $\sigma_{q}=0.01$.

and accurately measured with inexpensive equipment).

It is clear in all four runs that the velocity estimation using the Doppler information is substantially more accurate than what is provided by filtering and tracking with a position-only estimate. Further, the position tracking of the Doppler-aided estimates is more consistent in their accuracy and less susceptible to very large point estimation errors.

\section{Discussion}

Here we shall cover several results of the simulations and tests, their implications for tracker design, as well as other parts of the problem that are of interest.

\subsection{Node Failures}

In Section 2, we have assumed that all nodes in range have a perfect communication ability with the mobile node. In practice this might not be true, e.g., our pool tests experienced periodic node failures.

Node failure is allowed as long as there are enough measurements. Suppose that there are $N_{\text {eff }}$ successfully nodes, and order them as $n=1, \ldots, N_{\text {eff }}$, based on the order in which they 


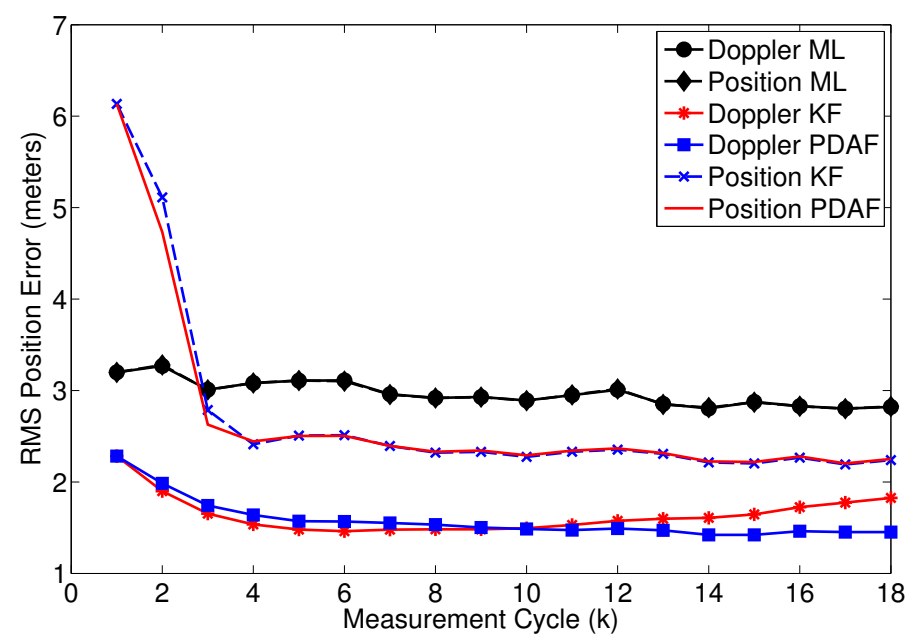

Figure 8: Comparison of simulated position error for the measurement, KF, and PDAF with $\sigma_{q}=0.1$.

(successfully) received their message from the mobile element. Equations (13) and (17) are still valid simply replacing $N$ by $N_{\text {eff }}$. Considering (17), in the 3-dimensional case, measurements from at least four nodes are needed. In the 2-dimensional case when the depth is known, measurements from at least three nodes are needed.

\subsection{Complexity of the Estimator}

The complexity of the presented maximum-likelihood algorithm via an exhaustive search would be $O\left(N L_{x} L_{y} L_{z}\right)$ for the position-only estimator, and $O\left(N L_{x} L_{y} L_{z} L_{\dot{x}} L_{\dot{y}} L_{\dot{z}}\right)$ for the complete estimator, where $L_{x}, L_{y}, L_{z}, L_{\dot{x}}, L_{\dot{y}}$, and $L_{\dot{z}}$ are the numbers of grid points for searching over the $x, y$, and $z$ coordinates and $\dot{x}, \dot{y}$, and $\dot{z}$ velocities, respectively, and $N$ is the number of participating anchor nodes. In practice, a coarse-grid search followed by a fine-grid search or a gradient-descent based search could be desirable.

\subsection{Target Tracker Selection}

Clearly the results of the simulations demonstrated that target tracking has a large potential to improve the overall position estimation. In the pool tests, this seemed largely to manifest as 


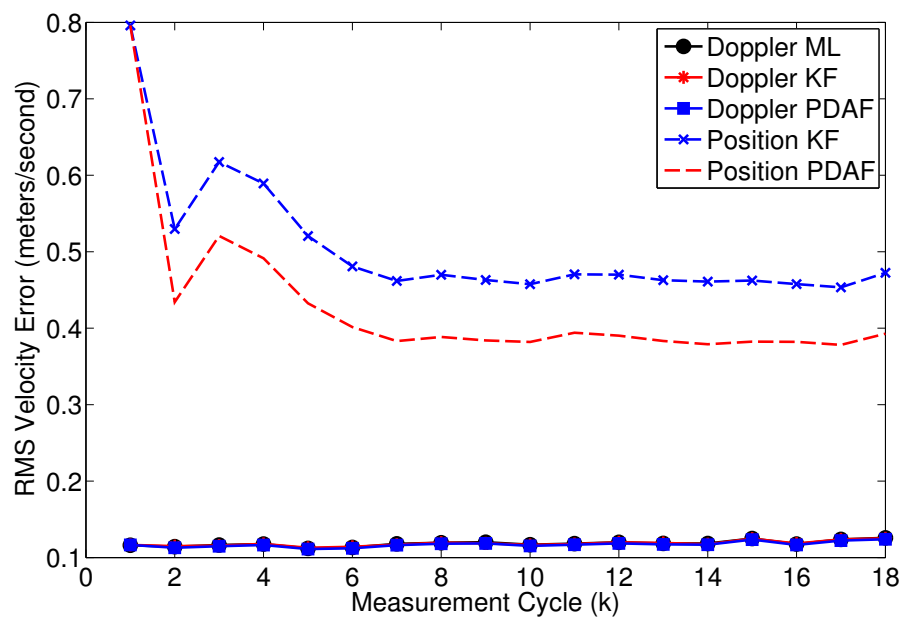

Figure 9: Comparison of simulated velocity error for the measurement, KF, and PDAF with $\sigma_{q}=0.1$.

as a smoothing of the error for the position estimator. This would indicate that there is some degree of model mismatch that might need to be addressed for field applications.

\subsection{Special Case of the Multistatic Tracker}

If we were to examine this problem considered in this paper as a range and range-rate tracking problem, it could be viewed as a special case of the multistatic target tracking problem where the transmitter is located at the target and we can ignore the presence of clutter. From there one could directly track the evolution of the target state from the range and range rate in a non-linear fashion without a measurement conversion.

The reason we excluded this result was two-fold: poor multiple measurement methods and batch measurements. Most of the methods for combining multiple measurements from the same target at the same time instant were drastically inferior to the multilateration ML we have derived and thus could not utilize as much information for a given time instant. The one that could have best served in handling this role was the maximum-likelihood probabilistic multiple-hypothesis tracker (ML-PMHT) [25]. However, when we relax the clutter assumption and derive the likelihood equations, it forms what is essentially a batch version of our current ML 


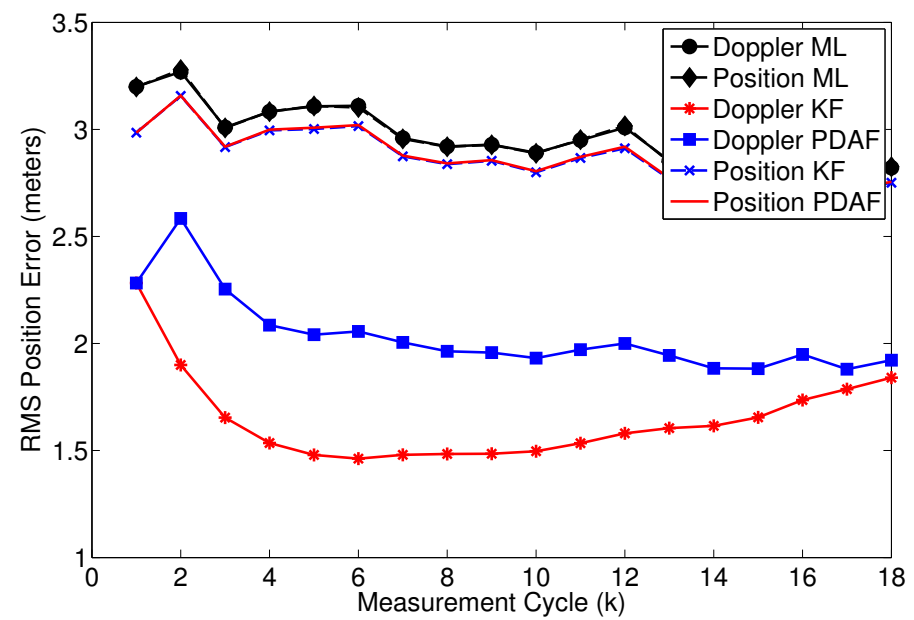

Figure 10: Comparison of simulated position error for the measurement, KF, and PDAF with $\sigma_{q}=1$.

multilateration estimate. Since our ML is already being supplemented with tracking algorithms, which is one attractive target-tracking option, we opted to not pursue an unconverted stateupdate.

\section{Conclusion}

In this work, we discussed the scenario of a moving unit communicating with several antenna nodes and estimating the position and velocity of that unit based on time-of-arrival measurements and Doppler speed estimates. A maximum likelihood estimator was derived, and several tracking filters were simulated and shown to offer an improvement over the individual point estimates in the context of a moving target. Pool testing concluded that the Doppler-aided approach does offer an performance improvement. Future work includes looking into computationally efficient implementations, better tracking and possible oceanic testing.

\section{Acknowledgement}

This work was partly supported by NSF grants ECCS-1310406 and IIS-1208499. P. Carroll was supported by a GAANN Fellowship from the Department of Education. K. Domrese was 


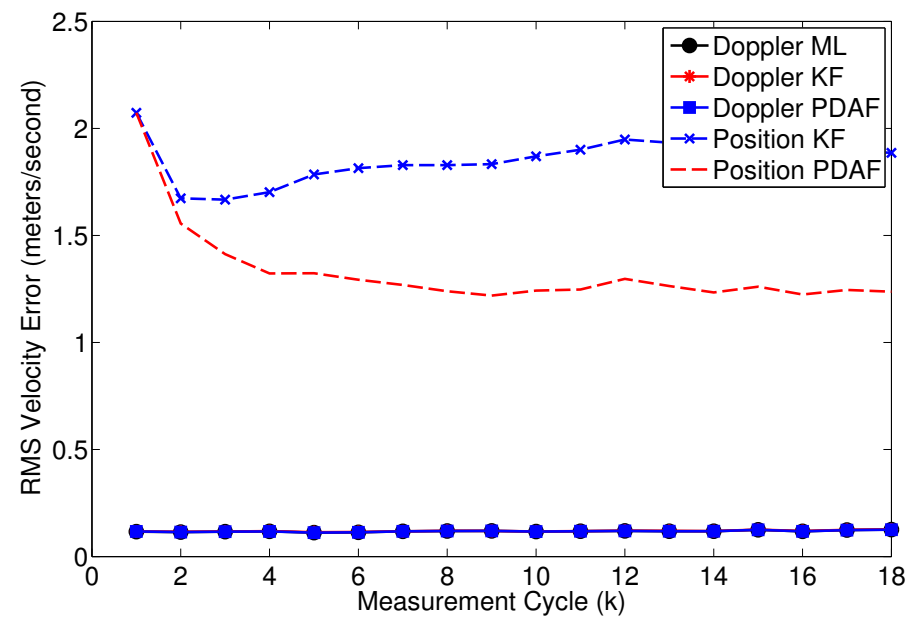

Figure 11: Comparison of simulated velocity error for the measurement, KF, and PDAF with $\sigma_{q}=1$.

supported by the NSF grant OCI-1156859 (REU site) and REU supplement. This work has been partially presented in the IEEE/MTS OCEANS conference, Taiwan, April 2014 [23] and the ACM WUWNET conference, Rome, Italy, November 2014 [26]. S. Zhou has an ownership interest in AquaSeNT, which is a marine sensor and communication technology firm that develops underwater wireless communication technology.

\section{References}

[1] Z.-H. Wang, S. Zhou, Z. Wang, J. Catipovic, and P. Willett, "Outage performance of a multiuser distributed antenna system in underwater acoustic channels," Proc. of the Asilomar Conference on Signals, Systems and Computers, Asilomar, California, Nov. 2012.

[2] S. Zhou, M. Zhao, X. Xu, J. Wang, and Y. Yao, "Distributed wireless communication system: A new architecture for future public wireless access," IEEE Communications Magazine, vol. 41, no. 3, pp. 108-113, Mar. 2003.

[3] R. Heath, T. Wu, Y. H. Kwon, and A. Soong, "Multiuser MIMO in distributed antenna 


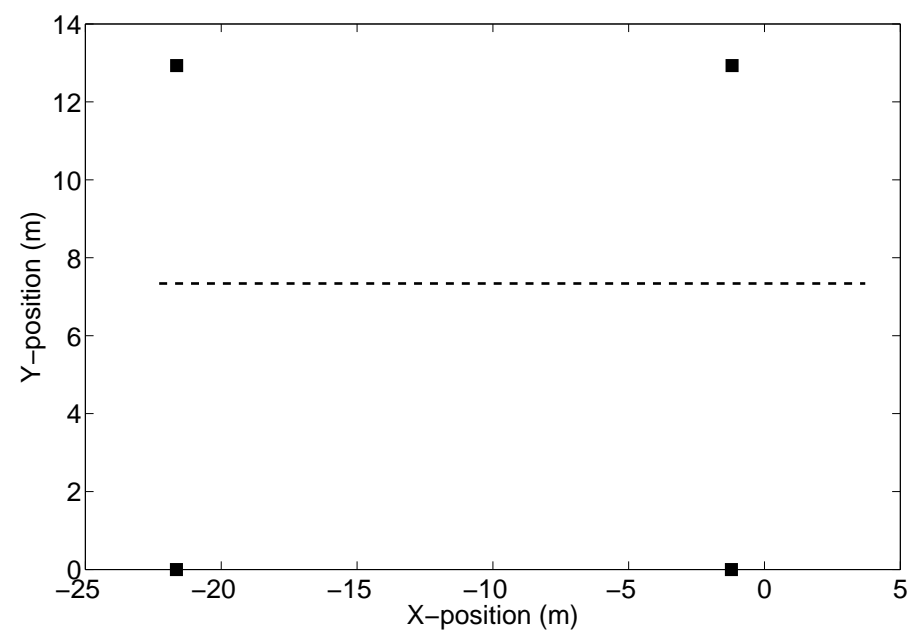

Figure 12: Pool layout for the test on June 24, 2014. Antenna nodes are denoted by the squares, and the general path of the transmitting node is denoted by the dashed line as it moved from left to right.

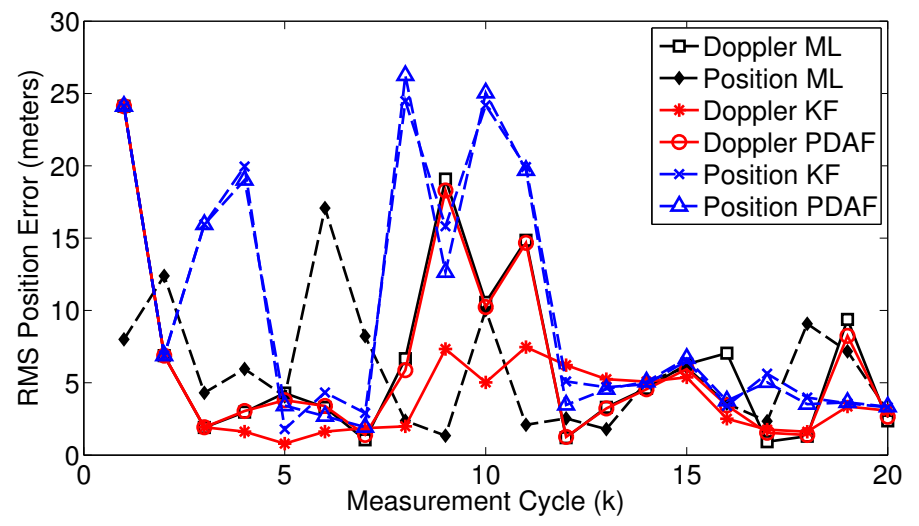

Figure 13: Comparison of position error for the measurement, KF, and PDAF for the first run during a pool test. 


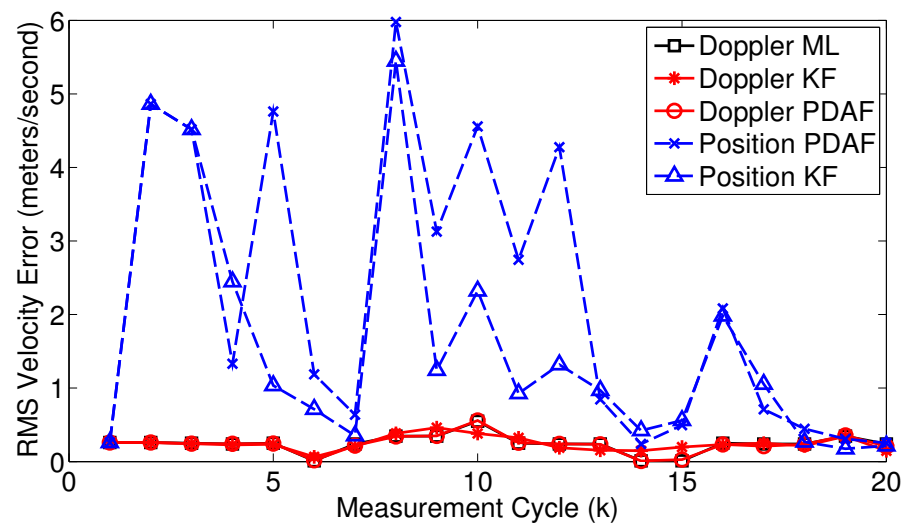

Figure 14: Comparison of velocity error for the measurement, KF, and PDAF for the first run during a pool test.

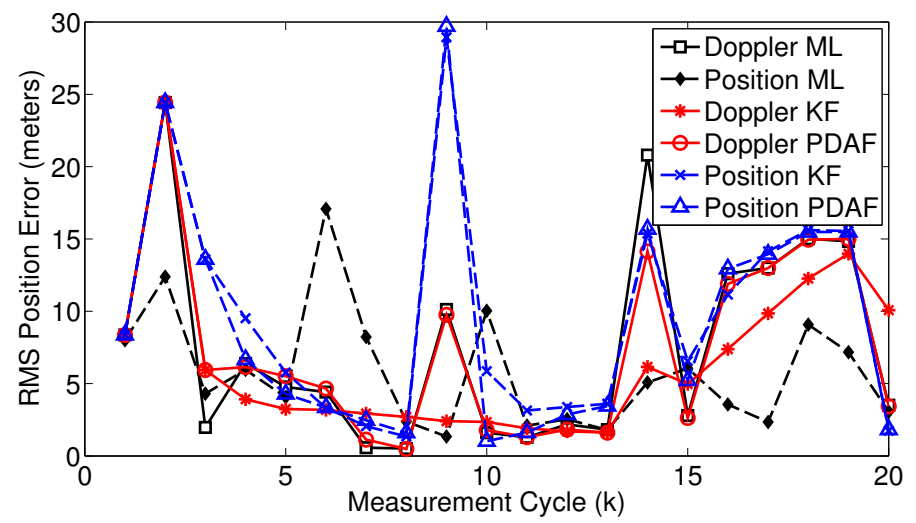

Figure 15: Comparison of position error for the measurement, KF, and PDAF for the second run during a pool test.

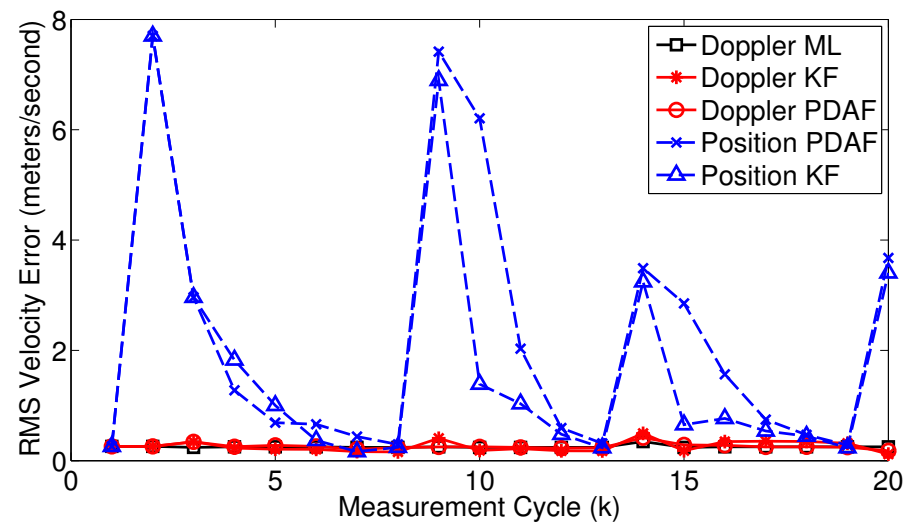

Figure 16: Comparison of velocity error for the measurement, KF, and PDAF for the second run during a pool test. 


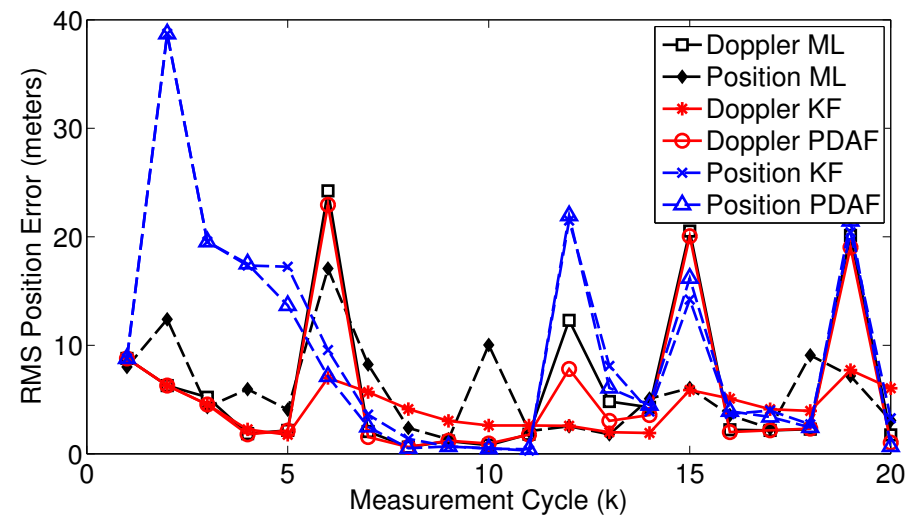

Figure 17: Comparison of position error for the measurement, KF, and PDAF for the third run during a pool test.

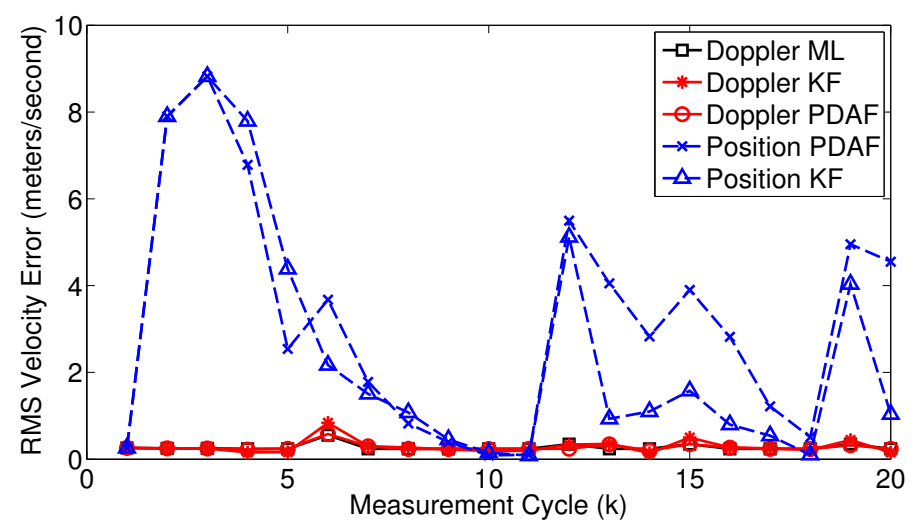

Figure 18: Comparison of velocity error for the measurement, KF, and PDAF for the third run during a pool test.

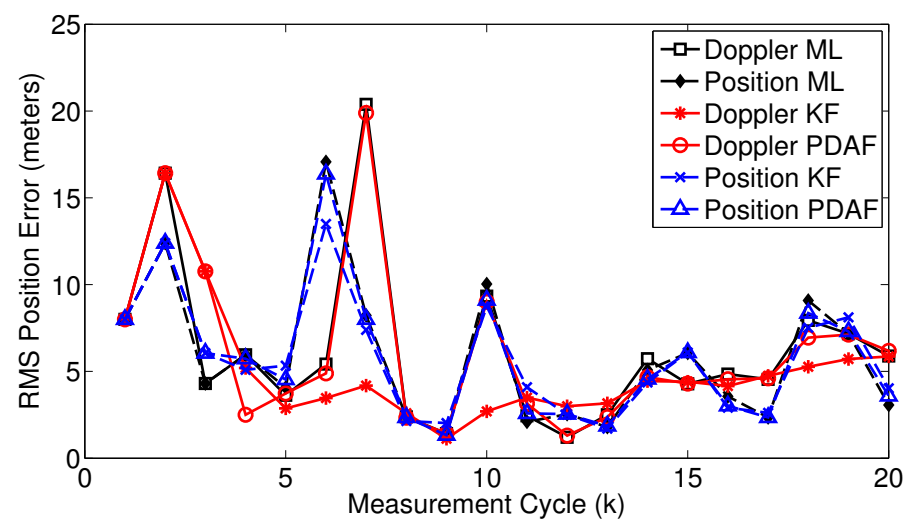

Figure 19: Comparison of position error for the measurement, KF, and PDAF for the fourth run during a pool test. 


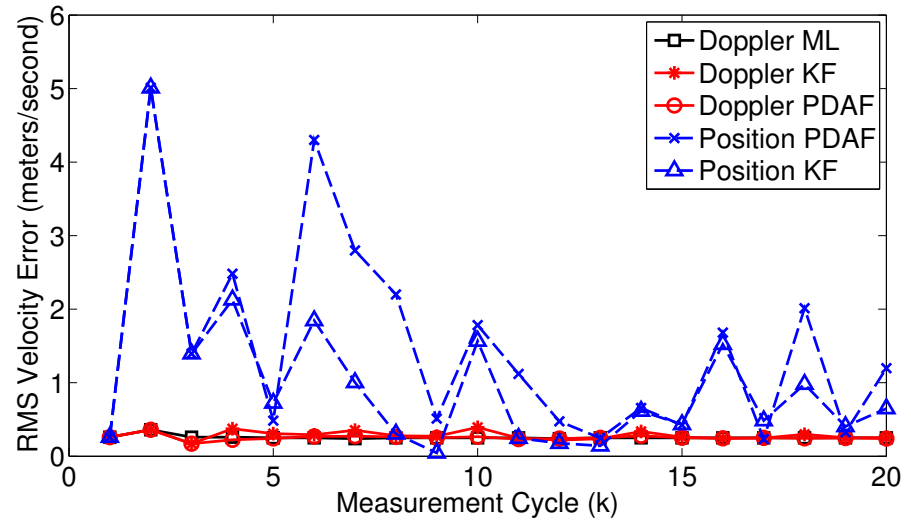

Figure 20: Comparison of velocity error for the measurement, KF, and PDAF for the fourth run during a pool test.

systems with out-of-cell interference," IEEE Trans. Signal Processing, vol. 59, no. 10, pp. 4885-4899, Oct. 2011.

[4] S.-R. Lee, S.-H. Moon, J.-S. Kim, and I. Lee, "Capacity analysis of distributed antenna systems in a composite fading channel," IEEE Trans. Wireless Commun., vol. 11, no. 3, pp. 1076-1086, Mar. 2012.

[5] R. Hayford, D. Nagle, and J. Catipovic, "AUTEC undersea cellular network," in Proc. of the ACM Intl. Workshop on Underwater Networks (WUWNet), Berkeley, California, Nov. 2009.

[6] H. P. Tan, R. Diamant, W. K. G. Seah, and M. Waldmeyer, "A survey of techniques and challenges in underwater localization," Ocean Engineering, vol. 38, no. 14-15, pp. 16631676, Oct. 2011.

[7] M. Erol-Kantarci, H. T. Mouftah, and S. Oktug, "A survey of architectures and localization techniques for underwater acoustic sensor networks," IEEE Communications Surveys $\&$ Tutorials, vol. 13, no. 3, pp. 487-502, third quarter 2011. 
[8] H. Tan, A. Gabor, Z. Eu, and W. Seah, "A wide coverage positioning system (WPS) for underwater localization," in Proc. of Intl. Conf. on Commun., May 2010.

[9] V. Chandrasekhar, W. K. Seah, Y. S. Choo, and H. V. Ee, "Localization in underwater sensor networks - survey and challenges," in Proc. First ACM International Workshop on Underwater Networks / MobiCom Conference, New York, NY, Sept. 2006, pp. 33-40.

[10] Z. Zhou, J.-H. Cui, and S. Zhou, "Efficient localization for large-scale underwater sensor networks," Ad Hoc Networks, vol. 8, pp. 267-279, 2010.

[11] D. Mirza and C. Schurgers, "Motion-aware self-localization for underwater networks," in Proc. of ACM International Workshop on Underwater Networks (WUWNet), San Francisco, California, USA, Sept. 2008, pp. 51-58.

[12] R. Diamant and L. Lampe, "Underwater localization with time-synchronization and propagation speed uncertainties," IEEE Trans. Mobile Computing, vol. 12, no. 7, pp. 1257-1269, 2013; doi=10.1109/TMC.2012.100.

[13] M. Erol, H. Mouftah, and S. Oktug, "Localization techniques for underwater acoustic sensor networks," IEEE Communications Magazine, vol. 48, no. 12, pp. 152-158, June 2010.

[14] B. Zhang, F. Yu, and Z. Zhang, "An improved localization algorithm for wireless sensor network using a mobile anchor node," in Proc. Asia-Pacific Conference on Information Processing (APCIP), vol. 2, July 2009.

[15] V. Chandrasekhar and W. Seah, "An area localization scheme for underwater sensor networks," Proc. of MTS/IEEE Oceans Conference, May 2007.

[16] Y. Wang, X. Ma, and G. Leus, "Robust time-based localization for asynchronous networks," IEEE Trans. Signal Processing, vol. 59, no. 9, pp. 4397-4410, 2011. 
[17] S. Chepuri, R. Rajan, G. Leus, and A.-J. van der Veen, "Joint clock synchronization and ranging: Asymmetrical time-stamping and passive listening," IEEE Signal Processing Letters, vol. 20, no. 1, pp. 51-54, Jan. 2013.

[18] M. Erol, L. F. M. Vieira, A. Caruso, F. Paparella, M. Gerla, and S. Oktug, "Multi stage underwater sensor localization using mobile beacons," in Proc. of the IEEE SENSORCOMM, pp. 710-714, Aug. 2008.

[19] P. Carroll, S. Zhou, H. Zhou, X. Xu, J.-H. Cui, and P. Willett, "Underwater localization and tracking of physical systems," Journal of Electrical and Computer Engineering, Special Issue on Underwater Communications and Networks, 2012, doi:10.1155/2012/683919.

[20] P. Carroll, K. Mahmood, S. Zhou, H. Zhou, X. Xu, and J.-H. Cui, "On-demand asynchronous localization for underwater sensor networks," IEEE Transactions on Signal Processing, vol. 62, no. 13, pp. 3337-3348, July 2014.

[21] B. Li, S. Zhou, M. Stojanovic, L. Freitag, and P. Willett, "Multicarrier communication over underwater acoustic channels with nonuniform Doppler shifts," IEEE Journal of Oceanic Engineering, vol. 33, no. 2, pp. 198-209, Oct. 2008.

[22] L. Wan, Z.-H. Wang, S. Zhou, T. Yang, and Z. Shi, "Performance comparison of Doppler scale estimation methods for underwater acoustic OFDM," Journal of Electrical and Computer Engineering, Special Issue on Underwater Communications and Networks, 2012, doi:10.1155/2012/703243.

[23] H. Gao, X. Xu, D. Huang, C.-F. Huang, T. Yang, W.-H. Twan, J.-Y. Liu, and S. Zhou, "A sea test of mobile underwater localization," in Proc. of IEEE/MTS OCEANS conference, Taipei, Taiwan, April 7-10, 2014.

[24] Y. Bar-Shalom, X. R. Li, and T. Kirubarajan, Estimation with Applications to Tracking and Navigation. New York: John Wiley \& Sons, 2001. 
[25] Y. Bar-Shalom, P. Willett, and X. Tian, Tracking and Data Fusion: A Handbook of Algorithms. YBS Publishing, 2011.

[26] P. Carroll, K. Domrese, H. Zhou, S. Zhou, and P. Willett, "Localization of mobile nodes in an underwater distributed antenna system," in Proc. of the 9th ACM International Conference on Underwater Networks \& Systems (WUWNet), Rome, Italy, Nov. 12-14, 2014. 


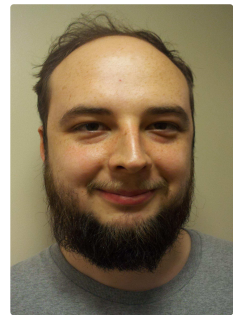

Patrick Carroll received his B.S. degree in 2009 and his M.S. degree in 2011, from the Department of Electrical and Computer Engineering, University of Connecticut (UCONN), Storrs, USA, both in electronic engineering. He recently completed his $\mathrm{PhD}$ degree in Electrical Engineering from UCONN.

His main research interests lie in the areas of network localization, navigation, and target tracking.

Katherine Domrese is currently an undergraduate student in the Department of Electrical and Computer

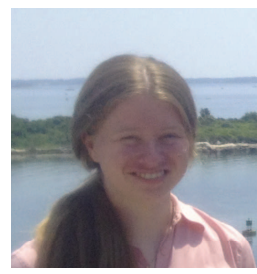

Engineering from the University of Connecticut, Storrs, CT, USA.

Her research interests include underwater acoustic communications, underwater localization, underwater robotics, and wireless sensor networks.

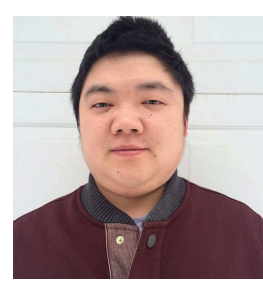

Hao Zhou received the B.S. degree in 2004 and the Ph.D degree in 2009, from the University of Science and Technology of China (USTC), Hefei, China, both in electronic engineering. After being a postdoctoral researcher in the Department of Electrical and Computer Engineering at the University of Connecticut (UCONN), he is now with Aquatic Sensor Network Technology (AquaSeNT).

His main research interests lie in the areas of communication systems, digital signal processing. His current focus is on the implementation of OFDM underwater acoustic modem.

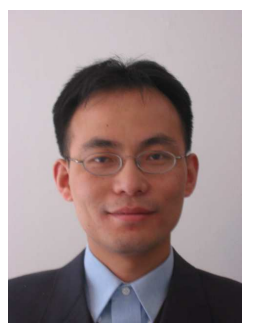

Shengli Zhou (F'14) received the B.S. degree in 1995 and the M.Sc. degree in 1998, from the University of Science and Technology of China (USTC), Hefei, both in electrical engineering and information science. He received his Ph.D. degree in electrical engineering from the University of Minnesota (UMN), Minneapolis, in 2002.

He is now a full professor with the Department of Electrical and Computer Engineering at the University of Connecticut (UCONN), Storrs. His general research interests lie in the areas of wireless communications and signal processing. His recent focus is on underwater acoustic communications and networking.

Dr. Zhou served as an associate editor for IEEE Transactions on Wireless Communications, Feb. 2005 - Jan. 2007, and IEEE Transactions on Signal Processing, Oct. 2008 - Oct. 2010. He is now an associate editor for IEEE Journal of Oceanic Engineering. He received the 2007 ONR Young Investigator award and the 2007 Presidential Early Career Award for Scientists and Engineers (PECASE). 


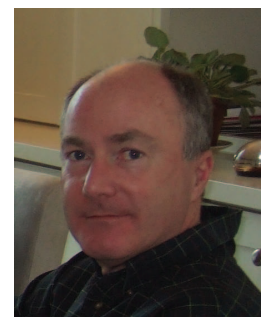

Peter Willett (F'03) received his BASc (Engineering Science) from the University of Toronto in 1982, and his PhD degree from Princeton University in 1986.

He has been a faculty member at the University of Connecticut ever since, and since 1998 has been a Professor. His primary areas of research have been statistical signal processing, detection, machine learning, data fusion and tracking. He has interests in and has published in the areas of change/abnormality detection, optical pattern recognition, communications and industrial/security condition monitoring.

Dr. Willett is editor-in-chief for IEEE Transactions on Aerospace and Electronic Systems, and until recently was associate editor for three active journals: IEEE Transactions on Aerospace and Electronic Systems (for Data Fusion and Target Tracking) and IEEE Transactions on Systems, Man, and Cybernetics, parts A and B. He is also associate editor for the IEEE AES Magazine, editor of the AES Magazines periodic Tutorial issues, associate editor for ISIF's electronic Journal of Advances in Information Fusion, and is a member of the editorial board of IEEE Signal Processing Magazine. He has been a member of the IEEE AESS Board of Governors since 2003. He was General Co-Chair (with Stefano Coraluppi) for the 2006 ISIF/IEEE Fusion Conference in Florence, Italy, Program Co-Chair (with Eugene Santos) for the 2003 IEEE Conference on Systems, Man, and Cybernetics in Washington DC, and Program Co-Chair (with Pramod Varshney) for the 1999 Fusion Conference in Sunnyvale. 